Amy VanScoy

University at Buffalo, Buffalo, NY, USA

\title{
RECONCILING DIFFERENT APPROACHES TO REFERENCE AND INFORMATION SERVICE USING Q METHDOLOGY (Paper)
}

\author{
Abstract or Résumé: \\ To make sense of the variety of conflicting conceptualizations of reference and information \\ service (RIS) in the literature, this study uses Q methodology, which involves a card sort and \\ interview, to identify librarians' espoused conceptualizations. Librarians in South Africa and the \\ United States sorted 35 statements representing the breadth of approaches to RIS. Preliminary \\ data were analyzed to identify prominent conceptualizations based on like sorts. Results will \\ inform theoretical work and professional education for RIS.
}

\section{Introduction}

In the literature, RIS has been conceptualized in a variety of ways. RIS scholars have mainly conceptualized it as information provision (e.g., Schiller 1965) or instruction (e.g., Elmborg 2002) or a pragmatic compromise between the two (Wyer 1930). Other RIS scholars have conceptualized it as an act of communication (e.g., Dervin \& Dewdney 1986, Radford 1996). Others have emphasized the essentially interpersonal nature of RIS (e.g., Cavanagh 2006, 2013, Radford \& Radford 2017). Other conceptualizations from the literature include counseling and partnering (VanScoy 2012). Practitioners, too, offer diverse perspectives on RIS when describing the work that they do (e.g., VanScoy 2016). To make advances in theoretical work and to adequately prepare future professionals for RIS, the discipline must reconcile these 
competing perspectives. Perhaps the single approaches taken by some scholars are oversimplified, and a combination approach, such as that taken by Radford and Radford, is needed. Perhaps the approaches described in the literature and not nuanced enough to adequately describe the phenomenon of RIS. This study uses Q methodology to make sense of the myriad and conflicting conceptualizations of RIS in the literature, responding to the following questions:

- How do experienced librarians conceptualize RIS?

- To what extend do librarians' espoused conceptualizations of RIS match those identified in the literature?

- How do librarians' conceptualizations of RIS differ by institutional context?

Decades of research in other disciplines, such as education, counseling, nursing and social work, have demonstrated the value of identifying conceptualizations of practice, using them to develop theory, improve practice, and guide professional education for pre-service practitioners (eg., Berman-Rossi 1988; Cook, Gilmer \& Bess 2003; Murray \& Macdonald 1997; Spruill \& Benshoff 2000). RIS could similarly benefit.

\section{Methodology}

In this study, Q methodology is used to examine conceptualizations of RIS by experienced librarians. The aim of Q methodology is to explore points of view or opinions. Brown (1993) describes it as the "systematic study of subjectivities" (93). Data is factored on clusters of opinions, rather than on individuals, allowing researchers to characterize perspectives about a phenomenon (Stephenson 1935; Watts \& Stenner 2005). Davies (1985) and Cushing (2017) have explored the method for research in library and information science (LIS). Q methodology has been used to study a wide range of LIS topics, including personal privacy on 
the web (Lee 2000), preservation of digital possessions (Cushing 2011), and the information search process (Denison \& Montgomery 2012).

The 70 participants in the study are drawn from Slovenia, South Africa and the United States to provide for a more global perspective than one country alone and to allow for crosscultural analysis. Participants also represent different institutional contexts, including academic, public, school, and special libraries.

Following Q methodology procedures, data for this study were collected using a card sort and brief interview to allow participants to elaborate on their sorting. The cards that the participants sorted, called the "Q sample", consisted of 35 statements that reflect conceptualizations of RIS. These statements are lightly edited quotations from librarians that reflect the breadth of conceptualizations represented in the literature. The Q sample includes statements such as "My job is to teach", "It's all about giving the person the answer", and "I think of reference librarianship as being a human services occupation in some ways. It's a very psychological or social work kind of thing.” The Q sample was tested with an experienced librarian to ensure that the statements did not contain more than one conceptualization and to ensure that the breadth of conceptualizations were represented (Paige \& Morin 2016). As a result of this procedure some of the statements were revised, such as the deletion of the phrase "in the community college" which would have made the statement specific to only one environment. Participants sorted the Q sample indicating those that are "most like how I think" and "least like how I think". Participants were then asked to elaborate on their choices to aid in interpreting the findings. The card sort was recorded and the brief interview transcribed.

Data were analyzed quantitatively, using Ken-Q Analysis, a web app designed specifically for Q methodology (https://shawnbanasick.github.io/ken-q-analysis/), to identify 
clusters of similar conceptualizations or "categories of thought" (Brown 1993, 120). Interview data were analyzed qualitatively to contribute to interpretation of the profiles generated by the web app.

\section{Results}

This study is currently a work in progress. Data from 35 participants of an expected 70 participants have been collected. Data collection is expected to be completed by the end of June 2019. The preliminary results presented here may have little resemblance to the final results. However, they illustrate the kind of findings that Q methodology will generate from this data.

The three factor solution chosen for the preliminary data has the advantage of strong loadings on each factor and statistically significant differences in the sorts, but it may be ignoring subtle nuances of opinion that could be more obvious in the final data set. For each factor, there are certain statements that best represent the factor, either positively or negatively. The distinguishing statements for each factor were examined to determine the characteristics of the conceptualization that each factor represents. These distinguishing statements have the most extreme factor loadings. Table 1 shows the highest ranked distinguishing statements for each Factor.

Table 1. Three highest ranked distinguishing statements for each factor. Factor Distinguishing Statements

Factor 1 The most important thing is that you're understanding what they're asking you. You have to be very clear to them. Words are a way to communicate, and they can also create a barrier if you're not receiving it in the same way.

Directing people to something... The role I play to them is like the pathfinder or the guide to what they want to know.

I really like the complex searching. 
Factor 2 When people ask a reference question, they're being a little bit vulnerable. They're acknowledging that they can't do something, and when people are vulnerable they need to trust the person they're addressing. They're kind of exposing themselves.

Providing a safe space and being a safe person for people to be vulnerable to as questioners, and I can be that with them.

I think of reference librarianship as being a human services occupation in some ways. It's very a psychological or social work kind of thing.

Factor 3 Ideally we don't want to hand them the answer, we want to empower them to find those answers, to be able to walk away and replicate what we've shown them.

My job is to teach.

A good reference interaction for me is that they learn the information literacy skills.

Participants from South Africa and the United States were distributed fairly evenly among the Factors. No participants working in school libraries loaded on Factor 1, and no participants working in special libraries loaded on Factor 2.

\section{Discussion}

Qualitative data from the brief interview are used to interpret the factors. The interpretation of the factors illustrate participants' conceptualizations of reference and information service based on clustering of like card sorts. This study is currently a work in progress, but it is clear that identifying factors helps to reconcile the variety of approaches that participants have toward reference and information service.

\section{Factor 1: Listening and information provision}

Participants who loaded on this factor highly ranked cards that referred to understanding the question, listening, and developing a rapport. In addition, they highly ranked cards referring 
to complex searching, identifying databases and keywords, and giving precise and accurate information. This finding shows that librarians from two cultures and all library environments emphasize the value of information provision and effective communication during the reference interaction.

\section{Factor 2: Counseling}

Participants who loaded on this factor highly ranked cards that referred to user vulnerability when asking questions, trust, the need to provide a safe space during the interaction, and the conceptualization of RIS as a human services occupation. They sorted cards relating to providing answers in a lower ranking. This Factor relates to the RIS literature relating to the interpersonal aspects of RIS. However, it more closely links to the scant literature conceptualizing RIS as Counseling (e.g., Maxfield,1954, Penland,1970), suggesting that this approach deserves greater attention in research and this literature deserves greater attention in professional education.

\section{Factor 3: Instruction}

Participants who loaded on this factor highly ranked statements that related to empowering users to find their own information, teaching, and learning information literacy skills. These participants also highly ranked the card describing librarians as an "agent of change". This finding supports the literature emphasizing the importance of RIS as a one-on-one instructional opportunity.

\section{Conclusion}

Initial data collection shows promise for Q methodology as a way of probing how librarians make sense of the differing conceptualizations of RIS in the literature. The preliminary results of the study support existing conceptualizations in the literature, focused on 
communication and information provision, counseling, and instruction. In addition, they reveal that librarians do have distinctive approaches to their work. Furthermore, these distinctions cross boundaries of culture and, with two notable exceptions, library environment.

Despite some exclamations during sorting of "This is hard!" and "This is painful!", participants have commented that the card sort method facilitates reflection and helps them identify key values in their practice.

With only half the data collected, these preliminary analyses may lack the depth and nuance that the full findings could offer. However, they demonstrate the differing conceptualizations of RIS in the literature exist in practice. A more nuanced understanding of RIS will help bridge the boundaries of theory and practice, providing conceptualizations of RIS grounded in practice and useful for theoretical work and professional education in RIS.

\section{Reference List:}

Berman-Rossi, T. (1988). Theoretical orientations of social work practice teachers: An analysis. Journal of Social Work Education, 24(1), 50-59.

Brown, S. R. (1993). A primer on Q methodology. Operant Subjectivity, 16(3/4), 91-138.

Cavanagh, M. (2006). Re-conceptualizing the 'reference transaction' - the case for interaction and information relationships at the public library reference desk. Canadian Journal of Information Studies, 30(1/2), 1-19.

Cavanagh, M. F. (2013). Interpreting reference work with contemporary practice theory. Journal of Documentation, 69(2), 214-242.

Cook, T. H., Gilmer, M. J., \& Bess, C. J. (2003). Beginning students' definitions of nursing: an inductive framework of professional identity. Journal of Nursing Education, 42(7), 311317. 
Cushing, A. L. (2011). Self-extension and the desire to preserve digital possessions. Proceedings of the American Society for Information Science and Technology, 48(1), 1-3.

Cushing, A. L. (2017). Applying Q method to archival research: A quantitative approach to qualitative study. In A. J. Gilliland, S. McKemmish \& A. J. Lau (Eds.) Research in the Archival Multiverse (1028-1052) Victoria, Australia: Monash University.

Davies, R. (1985). Q-analysis: A methodology for librarianship and information science. Journal of Documentation, 41(4), 221-246.

Denison, D. R., \& Montgomery, D. (2012). Annoyance or delight? College students' perspectives on looking for information. The Journal of Academic Librarianship, 38(6), 380-390.

Dervin, B., \& Dewdney, P. (1986). Neutral questioning: A new approach to the reference interview. $R Q, 25(4), 506-513$.

Elmborg, J. K. (2002). Teaching at the desk: Toward a reference pedagogy. portal: Libraries and the Academy, 2(3), 455-464.

Lee, B. (2000). Users' perspective on regulation to protect privacy on the web. The International Information \& Library Review, 32(3-4), 379-402.

Maxfield, D. K. (1954). Counselor librarianship: A new departure. Occasional Papers, 38, 1-39.

Murray, K., \& Macdonald, R. (1997). The disjunction between lecturers' conceptions of teaching and their claimed educational practice. Higher Education, 33(3), 331-349.

Paige, J. B., \& Morin, K. H. (2016). Q-sample construction: A critical step for a Qmethodological study. Western Journal of Nursing Research, 38(1), 96-110.

Penland, P. R. (1970). Interviewing for counselor and reference librarians. Pittsburgh: University of Pittsburgh. 
Radford, M. L. (1996). Communication theory applied to the reference encounter: An analysis of critical incidents. The Library Quarterly, 66(2), 123-137.

Radford, M. L., \& Radford, G. P. (2017). Library Conversations: Reclaiming Interpersonal Communication Theory for Understanding Professional Encounters. Chicago: American Library Association.

Schiller, A. R. (1965). Reference service: Instruction or information. The Library Quarterly, 35(1), 52-60.

Spruill, D. A., \& Benshoff, J. M. (2000). Helping beginning counselors develop a personal theory of counseling. Counselor Education and Supervision, 40(1), 70-80.

Stephenson, W. (1935). Correlating persons instead of tests. Journal of Personality, 4(1), 17-24.

VanScoy, A. (2012). Inventing the future by examining traditional and emerging roles for reference librarians. In M.L. Radford (Ed.), Leading the Reference Renaissance: Today's Ideas for Tomorrow's Cutting-Edge Services (pp. 79-94). New York: Neal-Schuman.

VanScoy, A. (2016). Making sense of professional work: Metaphors for reference and information service. Library \& Information Science Research, 38(3), 243-249.

Watts, S., \& Stenner, P. (2005). Doing Q methodology: theory, method and interpretation. Qualitative Research in Psychology, 2(1), 67-91.

Wyer, J. I. (1930). Reference Work: A Textbook for Students of Library Work and Librarians (Vol. 2). Chicago: American Library Association. 